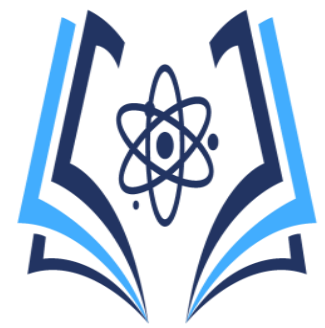

\title{
Covid 19 en adultos mayores con diabetes mellitus
}

\author{
Covid 19 in older adults with diabetes mellitus
}

Covid 19 en adultos mayores

\author{
Angélica María Quisiguiña Reyes Lcda. ${ }^{1}$ \\ Milton Espinoza Lucas MSc ICE ${ }^{2}$ \\ ${ }^{1}$ Universidad Estatal del Sur de Manabí, Jipijapa, Email: quisiguina-angelica9179@unesum.edu.ec \\ ${ }^{2}$ Universidad Laica Eloy Alfaro de Manabí, Manta, Email: espinoza-milton@unesum.edu.ec
}

Contacto: espinoza-milton@unesum.edu.ec

Recibido: 25-02-2020

Aprobado: 23-06-2020

\section{Resumen}

La Diabetes Mellitus es una enfermedad crónica relevante para el Ecuador, debido al aumento en su prevalencia de morbi-mortalidad, al igual que el nuevo virus de COVID 19 ha generado una problemática en cuanto a salud pública, no solo para nuestro país sino también a nivel mundial. El presente trabajo investigativo tiene como objetivo general determinar los factores asociados a complicación por Covid 19 en adultos mayores diabéticos de la ciudadela Parrales Y Guale en la ciudad de Jipijapa, evaluando el grado de exposición de contagio y manejo familiar. $\mathrm{La}$ metodología fue de tipo observacional-descriptivotransversal-prospectivo; empleando la encuesta de recolección de datos a los sujetos de investigación de manera directa o indirecta, los cuales tenían diagnósticos de diabetes mellitus y Covid 19. Los resultados indican que la comorbilidad más frecuente es la hipertensión con el 53\%, el $83 \%$ tuvieron síntomas leves, la sintomatología más común fue del $37 \%$ cefalea, el contagio con mayor frecuencia fue por contacto familiar con una proporción del $73 \%$, las glicemias reflejadas fueron de $125-200 \mathrm{mg} / \mathrm{dl}$ con el $70 \%$, el estado actual de los adultos mayores se encuentra en condiciones estables con el $57 \%$, el $16 \%$ se complicaron y fallecieron. Se concluye que los pacientes encuestados presentan alto nivel de riesgo de complicación de la enfermedad basal, aumentando la mortalidad en un manejo tardío de las complicaciones, por lo que los cuidados preventivos son importantes para el autocuidado de la población y que estos están predispuestos a conocer y aplicarlos para salvaguardar su vida.

Palabras claves: Diabetes, complicaciones, prevención, tratamiento

\begin{abstract}
Diabetes Mellitus is a relevant chronic disease for Ecuador, due to the increase in its prevalence of morbidity and mortality, just as the new COVID 19 virus has generated a problem in terms of public health, not only for our country but also for world level. The general objective of this research work is to determine the factors associated with complications from Covid 19 in diabetic older adults from the Parrales Y Guale in the city of Jipijapa, evaluating the degree of exposure to contagion and family management. The methodology was observational-descriptivetransversal-prospective; using the data collection survey to the research subjects directly or indirectly, who had diagnoses of diabetes mellitus and Covid 19. The results indicate that the most frequent comorbidity is hypertension with $53 \%$, $83 \%$ had mild symptoms, the most common symptomatology was $37 \%$ headache, the most frequent contagion was by family contact with a proportion of $73 \%$, glycemic reflected was $125-200$ $\mathrm{mg} / \mathrm{dl}$ with $70 \%$, the current state of the elderly is in stable condition with $57 \%, 16 \%$ became complicated and died. It is concluded that the surveyed patients present a high level of risk of complication of the baseline disease, increasing
\end{abstract}




\section{HIGÍA DE LA SALUD}

mortality in late management of complications, so preventive care is important for the self-care of the population and that they are predisposed to know and apply them to safeguard your life.

Keywords: Diabetes, complications, prevention, treatment

\section{Introducción}

En la actualidad, es necesario conocer el impacto de la Covid 19 ante la sociedad y tener claro el potencial riesgo que conlleva, sin distinción de etnia, edad o género, por ende las poblaciones vulnerables será la más afectada. (1)

Las poblaciones vulnerables son aquellos grupos de personas, que comparten una posibilidad mayor de ser dañados ya sea por ausencia o falta de desarrollo adecuado de competencias, situaciones de empoderamiento habilidades o capacidades; con el fin de disminuir, atenuar o evitar el riesgo específico. (2)

La Covid 19 pertenece a una familia amplia de virus que principalmente provoca afectaciones leves del sistema respiratorio superior, por lo tanto sus mutaciones de los receptores de superficie han producido infecciones graves de la parte inferior del sistema respiratorio, como el Síndrome Respiratorio Agudo Severo (SARS-CoV y el Síndrome Respiratorio del Medio Oriente (MERSCoV) y) (3)

El nacimiento de la pandemia por COVID-19 supone un reto de enormes dimensiones, y la gran presencia de diabetes mellitus tipo 2 en la población actual hace que sea un problema de salud en el que se debe centrar el esfuerzo para dar la mejor respuesta a los pacientes que son más vulnerables al desarrollo de la infección y se convierten en candidatos a presentar cuadros clínicos más graves.

En la actualidad, una nueva clase de coronavirus está causando epidemias de enfermedades graves y muerte en humanos. Para abordar la relación entre la infección por COVID-19 y la Diabetes Mellitus Tipo 2 en personas adultos mayores se debe analizar datos epidemiológicos que sustenten esta asociación y, posteriormente profundizar en los mecanismos fisiopatológicos que podrían conectar a ambas enfermedades.

Se reportaron una serie de neumonía atípica a finales de diciembre del 2019, en ese entonces no se conocía su origen. Posteriormente se identificó el agente etiológico al cual se le llamó SARSCOV-2 y la enfermedad que produce se la nombró
ISSN 2773-7705

Periodo. Enero - Junio 2020

Vol. 2, Nro. 1, Publicado 2020-06-30

COVID 19. El origen de este nuevo virus es zoonótico siendo los murciélagos el probable vector. Dada la propagación por el alto número de contagios e incremento de muerte pasó de ser un brote aislado a una emergencia sanitaria de gran preocupación mundial, en la actualidad una gran pandemia. (4)

Vale destacar en breve las principales pandemias que se dieron a inicios del siglo XXI: SARS (Síndrome Respiratorio Agudo Grave) iniciado en Cantón-China, 2002 , Gripe Aviaria (H5N1) iniciado en Bundang-Corea del Sur, 2003, Gripe Porcina (H1N1) iniciada en California -Estados Unidos, 2009 con mayor impacto en Argentina con 626 muertes, Fiebre Hemorrágica, Ébola, iniciada en Guinea -África, 2014, Zika iniciada en islas del Pacífico-Polinesia francesa, 2013 y la actual de COVID-19 iniciada en Wuhan-China, 2019 . La sede originaria del nuevo coronavirus se dio en Wuhan, la mega ciudad capital de la provincia de Hubei considerada el mayor núcleo poblacional de la zona central del país cuenta con aproximadamente 12 millones de habitantes. Siendo el principal centro político, económico y educativo de China central y un nodo principal de transportes que la vincula a todo el país. (5)

La Comisión Municipal de Salud reportó a la Organización Mundial de la Salud de un brote de veintisiete casos similares a la neumonía el 31 de diciembre de 2019, en primera instancia se afirmaba que el origen fue en el mercado de pescados y mariscos de Hua Nan, el 17 de noviembre por parte del gobierno chino se presenta el primer caso de Covid-19 y al menos 266 personas infectadas permaneciendo bajo vigilancia médica. Las autoridades de Wuhan negaban pasar por una crisis sanitaria similar al brote del SARS del año 2002-2003 tratando de ocultar lo que estaba sucediendo. Al finalizar las 2019 ocho personas ligadas al Hospital Central de Wuhan compartieran por red social de Weibo algunas evidencias respecto a la manera en que varios pacientes mostraban un rápido deterioro y fallas graves en sus sistemas respiratorios. La policía de Wuhan afirmó el 1 de enero de 2020 tomar medidas legales contra estas personas por divulgar rumores, cinco días después se confirmó la existencia de pacientes con neumonía de origen a un virus distinto, desconociendo su morbilidad, velocidad de expansión y letalidad. (6)

El 11 de marzo de 2020 debido a la emergencia sanitaria desencadenada por la Covid 19, la Organización Mundial de la Salud anunció que es una "emergencia de salud pública de importancia 


\section{HIGÍA DE LA SALUD}

internacional". Los primeros brotes de transmisión local se dieron en Italia, produciendo una masiva ola de casos, representando un foco importante de contagio; con ello se aplicó confinamiento a toda esta región junto con otras 14 provincias del norte de Italia. Al final del mes de marzo la pandemia ya se había extendido a 190 países, a pesar de las medidas de contención, cierres parciales o completos de fronteras y a restricciones para viajar. En muchos países, estas medidas fueron muy estrictas, pero a pesar de ello al finalizar ese mes los registros oficiales ya contabilizaban un total mundial de 3,23 millones de contagio y 215.000 muertes, al comenzar el mes de mayo la fase ascendente de la enfermedad apenas comenzaba en África frágil y vulnerable debido al sistema de salud que posee, por otra parte, se reflejaba aún más los contagios en Estados Unidos y Brasil. (7)

En nuestro país el primer caso registrado fue el 29 de febrero del 2020 siendo una mujer de 71 años que retornó de España el 14 de febrero y el 13 de marzo se notificó su deceso. Los casos de contagio y fallecidos seguían aumentando, con lo cual el mandatario aplicó toque de queda como medida restrictiva. (8). En el Ecuador los contagios empezaron a ser más altos al décimo día de haber reportado el primer caso, a nivel nacional la tasa de infección por coronavirus tiene un comportamiento diferente en cada provincia, siendo Guayas, Galápagos y Cañar que poseen valores más altos. (9)

La diabetes es una patología prevalente durante la pandemia y se ha identificado como un factor de riesgo para desarrollar un cuadro grave de COVID 19, es un gran desafío para las personas que viven con esta patología y sobre todo para el personal de salud asistencial, ya que estos pacientes necesitan cuidados y atención especial, pues su enfermedad está relacionada con una mayor agravación de síntomas y por ende complicaciones. Entendemos que la diabetes es una enfermedad crónica no transmisible conocida como no insulinodependiente a razón de una utilización ineficaz de la insulina, la mayor parte de los casos se debe al sobrepeso y falta de actividad física. (10)

\section{Materiales y métodos}

Para la investigación se consideró el sector urbano ciudadela Parrales Y Guale de la ciudad de Jipijapa-Manabí-Ecuador, se aplicó la técnica de muestreo probabilístico de los casos identificados en la comunidad, de los cuales se obtuvo una muestra de 30 pacientes adultos mayores diabéticos con diagnóstico confirmado de Covid 19.
ISSN 2773-7705

Periodo. Enero - Junio 2020

Vol. 2, Nro. 1, Publicado 2020-06-30

El porcentaje de número de personas diabéticas en las últimas tres décadas a nivel mundial se ha cuadriplicado convirtiéndose en la novena causa de muerte, según estudios afirman que 1 de cada 11 individuos a nivel mundial la posee; aproximadamente el $90 \%$ de los casos se diagnostican con diabetes mellitus tipo 2, centrándose como epidemia global en Asia, China e India. (11)

De acuerdo a la Organización Mundial de la Salud, se estima que alrededor de 62,8 millones de personas en las Américas padece de diabetes; el número de personas con diabetes en América Latina podría subir de 25 a 40 millones para el año 2030. La doctora Roopa Mehta, miembro del sistema nacional de investigadores de México, dio a conocer que la prevalencia de diabéticos no insulinodependiente podrían incrementar hasta un $62 \%(12)$

Según encuestas con instrumentación STEP realizadas en el 2018 para medir factores de riesgo de las enfermedades crónica no transmisibles, en el país el $7,8 \%$ de la población padece de hiperglucemia incrementando el riesgo de desarrollar diabetes mellitus. La diabetes es considerada la segunda causa de muerte de acuerdo al Instituto Nacional de Estadística y Censo (INEC) entre los años 2014 y 2017 y además en el año 2016 a 2017 las personas fallecidas por diabetes fueron de 4895. (13)

El objetivo general es determinar los factores asociados a complicación por Covid 19 en adultos mayores diabéticos de la ciudadela Parrales Y Guale en la ciudad de Jipijapa., teniendo como objetivos específicos identificar la frecuencia de adultos mayores diabéticos con Covid 19, medir los factores biológicos, socio-sanitarios de los adultos mayores con Covid 19, determinar los marcadores de riesgo de los sujetos de investigación. La población de referencia son los pacientes adultos mayores diabéticos con diagnóstico confirmado de Covid 19.

Se aplicó el método cuantitativo con estudio tipo observacional- descriptivo-transversalprospectivo, empleando la herramienta de investigación para la recolección de datos la encuesta, adaptada de Quimis y Morales. Para la validación del estudio se utilizó el documento del STROBE para asegurar las características estructurales del informe. 
Los criterios de inclusión que se consideraron son personas con igual o mayor edad de 65 años, que poseen diagnóstico confirmado de Diabetes mellitus y Covid 19, que colaboran con el interrogatorio y firman su consentimiento para la investigación.

Dentro de los criterios de exclusión se consideran pacientes que no tienen pruebas confirmatorias de Covid 19 o que son diagnósticos como casos sospechosos o probables, además los que son considerados como contactos. Los criterios de eliminación son las familias donde no se
Vol. 2, Nro. 1, Publicado 2020-06-30

\section{TABLA 1. SEXO}

encontraron personas en sus casas o que no desearon colaborar con la investigación

La información fue bajo confidencialidad y consentimiento informado. La encuesta se aplicó directamente previa a la explicación de la herramienta que consta de 19 preguntas con lenguaje claro, entendibles encaminadas al logro de los objetivos planteados.

\section{Resultados}

Los resultados de las encuestas fueron tabulados y organizados teniendo como interpretación lo siguiente:

\begin{tabular}{lll}
\hline ALTERNATIVA & FRECUENCIA & PORCENTAJE \\
HOMBRE & 14 & $47 \%$ \\
MUJER & 16 & $53 \%$ \\
TOTAL & 30 & $100 \%$ \\
\hline
\end{tabular}

Fuente: Encuesta realizada en la Ciudadela PARRALES Y GUALE

Elaborado por: Autora

En la tabla 1 nos muestra que el sexo más encuestado corresponde a mujeres con el $53 \%$.

TABLA 2. EDAD

\begin{tabular}{lll}
\hline ALTERNATIVA & FRECUENCIA & PORCENTAJE \\
$65-75$ años & 22 & $74 \%$ \\
$75-85$ años & 7 & $23 \%$ \\
$85-95$ años & 1 & $3 \%$ \\
95 en adelante & 0 & $0 \%$ \\
TOTAL & 30 & $100 \%$ \\
\hline
\end{tabular}

Fuente: Encuesta realizada en la Ciudadela PARRALES Y GUALE

Elaborado por: Autora

En la tabla 2 indica los resultados según la edad comprobando que la mayor parte de las personas diabéticas encuestadas están entre las edades de 65 a 75 años con el $74 \%$.

TABLA 3. COMORBILIDAD

\begin{tabular}{lll}
\hline ALTERNATIVA & FRECUENCIA & PORCENTAJE \\
Hipertensión & 16 & $53 \%$ \\
Insuficiencia Renal & 2 & $7 \%$ \\
Otras & & $0 \%$ \\
Ninguna & 12 & $40 \%$ \\
TOTAL & 30 & $100 \%$ \\
\hline
\end{tabular}

Fuente: Encuesta realizada en la Ciudadela PARRALES Y GUALE

Elaborado por: Autora 
En la tabla 3 se muestra las comorbilidades adyacentes a la diabetes mellitus siendo la hipertensión arterial la más asociada con el $53 \%$.

TABLA 4. FRECUENCIA DE SÍNTOMAS

\begin{tabular}{lll}
\hline ALTERNATIVA & FRECUENCIA & PORCENTAJE \\
Leves & 25 & $83 \%$ \\
Graves & 5 & $17 \%$ \\
TOTAL & 30 & $100 \%$ \\
\hline
\end{tabular}

Fuente: Encuesta realizada en la Ciudadela PARRALES Y GUALE

Elaborado por: Autora

En la tabla 4 nos indica que los síntomas que presentaron los adultos mayores fueron leves en un $83 \%$.

TABLA 5. SINTOMATOLOGÍA

\begin{tabular}{lll} 
ALTERNATIVA & FRECUENCIA & PORCENTAJE \\
Dolor de cabeza & 11 & $37 \%$ \\
Dolor de garganta & 4 & $13 \%$ \\
Fiebre & 6 & $20 \%$ \\
Diarrea & 3 & $10 \%$ \\
Dificultad respiratoria & 5 & $17 \%$ \\
Perdida del gusto y olfato & 1 & $3 \%$ \\
Otros & 0 & $0 \%$ \\
TOTAL & 30 & $100 \%$ \\
\hline
\end{tabular}

Fuente: Encuesta realizada en la Ciudadela PARRALES Y GUALE

Elaborado por: Autora

En la tabla 5 se muestra los síntomas más frecuentes que el adulto mayor diabético experimentó durante la enfermedad por Covid 19; siendo el más común el dolor de cabeza con un $37 \%$, seguido de fiebre con un $20 \%$.

TABLA 6. CONTAGIO A COVID 19

\begin{tabular}{|c|c|c|c|}
\hline ALTERNATIVA & & FRECUENCIA & PORCENTAJE \\
\hline Trabajo & & 0 & $0 \%$ \\
\hline $\begin{array}{l}\text { Contacto Familiar } \\
\text { domiciliarias) }\end{array}$ & (visitas & 22 & $73 \%$ \\
\hline Adquisición de víveres & & 3 & $10 \%$ \\
\hline Contacto Social & & 0 & $0 \%$ \\
\hline Cita Médica & & 5 & $17 \%$ \\
\hline Viajes & & 0 & $0 \%$ \\
\hline TOTAL & & 30 & $100 \%$ \\
\hline
\end{tabular}

Fuente: Encuesta realizada en la Ciudadela PARRALES Y GUALE

Elaborado por: Autora

La tabla 6 indica el motivo más frecuente por el cual el adulto mayor se contagió a Covid-19 fue por contacto familiar con un $73 \%$. 
TABLA 7. ATENCIÓN SANITARIA

\begin{tabular}{lll}
\hline ALTERNATIVA & FRECUENCIA & PORCENTAJE \\
Público & 19 & $63 \%$ \\
Privada & 8 & $27 \%$ \\
Se automedicó & 3 & $10 \%$ \\
TOTAL & 30 & $100 \%$
\end{tabular}

Fuente: Encuesta realizada en la Ciudadela PARRALES Y GUALE

Elaborado por: Autora

En la tabla 7 se muestra que el $63 \%$ de los pacientes adultos mayores diabéticos recibieron atención pública pero es notable además que el $10 \%$ se auto medicó.

TABLA 8. SUSPENSIÓN DE TRATAMIENTO PARA DIABETES

\begin{tabular}{lll}
\hline ALTERNATIVA & FRECUENCIA & PORCENTAJE \\
SI & 3 & $10 \%$ \\
NO & 27 & $90 \%$ \\
TOTAL & 30 & $100 \%$ \\
\hline
\end{tabular}

Fuente: Encuesta realizada en la Ciudadela PARRALES Y GUALE

Elaborado por: Autora

La tabla 8 indica que el 10\% de adultos mayores diabéticos suspendieron tratamiento de base.

TABLA 9. MOTIVO DE ABANDONO DE TRATAMIENTO

$\begin{array}{lll}\text { ALTERNATIVA } & \text { FRECUENCIA } & \text { PORCENTAJE } \\ \text { Falta de medicina } & 3 & 10 \% \\ \text { Suspensión de cita } & 0 & 0 \% \\ \text { Miedo a salir y contagiarse } & 0 & 0 \% \\ \text { Decidió suspenderla } & 0 & 0 \% \\ \text { Cambio de medicamento por } & 0 & 0 \% \\ \text { medicina natural u otra alternativa } & & 90 \% \\ \text { Continuó con su tratamiento } & 27 & 100 \% \\ \text { TOTAL } & 30 & \end{array}$

Fuente: Encuesta realizada en la Ciudadela PARRALES Y GUALE

Elaborado por: Autora

En la tabla 9 se indica que el motivo más frecuente para abandonar el tratamiento para la diabetes fue por falta de medicamentos.

TABLA 10. FACTOR EMOCIONAL

\begin{tabular}{lll}
\hline ALTERNATIVA & FRECUENCIA & PORCENTAJE \\
$\begin{array}{l}\text { Se sintió bien por el cuidado de su } \\
\text { familiar }\end{array}$ & 5 & $17 \%$ \\
$\begin{array}{l}\text { Se sintió triste por pérdida de } \\
\text { familiar }\end{array}$ & 2 & $7 \%$ \\
$\begin{array}{l}\text { Permaneció solo } \\
\text { Preocupado por la pandemia }\end{array}$ & 0 & $0 \%$ \\
\cline { 2 - 3 } & 23 & $76 \%$
\end{tabular}

https://revistas.itsup.edu.ec/index.php/higia 
Fuente: Encuesta realizada en la Ciudadela PARRALES IGUALE

Elaborado por: Autora

En la tabla 10 el $76 \%$ de los adultos mayores se encuentran preocupados por la pandemia.

TABLA 11. HÁBITOS ALIMENTICIOS

\begin{tabular}{lll}
\hline ALTERNATIVA & FRECUENCIA & PORCENTAJE \\
Bebidas azucaradas & 0 & $0 \%$ \\
Comidas rápidas & 0 & $0 \%$ \\
Comidas enlatadas & 4 & $14 \%$ \\
Frituras & 0 & $0 \%$ \\
Carbohidratos & 5 & $17 \%$ \\
Carnes rojas & 1 & $3 \%$ \\
Pescado & 10 & $33 \%$ \\
Frutas y verduras & 10 & $33 \%$ \\
TOTAL & 30 & $100 \%$ \\
\hline
\end{tabular}

Fuente: Encuesta realizada en la Ciudadela PARRALES Y GUALE

Elaborado por: Autora

En la tabla 11 indica una buena dieta de hábitos alimenticios con un 33\% de pescado, frutas y verduras.

TABLA 12. VALORES DE GLICEMIA

\begin{tabular}{lll}
\hline ALTERNATIVA & FRECUENCIA & PORCENTAJE \\
$80-100 \mathrm{mg} / \mathrm{dl}$ & 0 & $0 \%$ \\
$100-125 \mathrm{mg} / \mathrm{dl}$ & 2 & $7 \%$ \\
$125-200 \mathrm{mg} / \mathrm{dl}$ & 21 & $70 \%$ \\
Mayor a $200 \mathrm{mg} / \mathrm{dl}$ & 4 & $13 \%$ \\
No conoce & 3 & $10 \%$ \\
TOTAL & 30 & $100 \%$ \\
\hline
\end{tabular}

Fuente: Encuesta realizada en la Ciudadela PARRALES Y GUALE

Elaborado por: Autora

En la tabla 12 las glicemias fueron altas con valores de $125-200 \mathrm{mg} / \mathrm{dl}$ que corresponden al $70 \%$ de $10 \mathrm{~s}$ adultos mayores

TABLA 13. ESTADO ACTUAL

\begin{tabular}{lll}
\hline ALTERNATIVA & FRECUENCIA & PORCENTAJE \\
Mejoró su estado de salud & 8 & $27 \%$ \\
Estable, pero presenta secuelas & 17 & $57 \%$ \\
Se complicó y falleció & 5 & $16 \%$ \\
TOTAL & 30 & $100 \%$ \\
\hline
\end{tabular}

Fuente: Encuesta realizada en la Ciudadela PARRALES Y GUALE

Elaborado por: Autora 
En la tabla 13 se muestra que la afección por Covid en este grupo etario más una comorbilidad dejó secuelas en la salud de la mayor parte de estos pacientes, pero además representa un considerable porcentaje que se complicaron y fallecieron.

\section{Discusión}

La diabetes mellitus es un problema de salud pública, ya que su incidencia aumenta con el pasar de los años elevando la tasa de morbilidad y mortalidad, esta patología desencadena un desequilibrio metabólico trayendo consigo complicaciones crónicas en el ser humano, en la actualidad con la presencia de un nuevo virus es preocupante la salud de los adultos mayores ya que la diabetes es un factor predisponente que empeora el estado de salud de estos pacientes.

Se presentan los hallazgos más relevantes del estudio, mostrando que el grupo afectado está en rangos de edades de 65 a 75 años, en comparación del artículo publicado por el ministro de salud púbica que reporta que el grupo poblacional con mayor incidencia es el de 20 a 49 años. (14).

El estudio de Ashkan y sus colaboradores manifiestan que las comorbilidades parecen ser un requisito previo para la infección por COVID 19, sintomática y grave, sin embargo, en nuestros resultados la mayor parte de adultos mayores tienen diabetes más hipertensión. (15)

El cuadro clínico de muchos estudios científicos, la diferencia de síntomas leves, moderados y graves, según el informe de la misión de la OMS en china, describen un cuadro amplio más sintomatología respiratoria, nuestro estudio mostro una triada de cefalea fiebre y dolor de garganta dando un síndrome de diagnósticos diferenciales. (16)

Una revisión rápida de literatura ha evaluado que el uso de un cubre boca y nariz independiente del material, tiene un efecto pequeño en la reducción en la transmisión comunitaria de virus respiratorios entre familiares, por los que los autores de la revisión consideran que en un espacio pequeño la transmisibilidad del virus es alta y apoyan el uso de la mascarilla, sin embargo en nuestro resultados el $73 \%$ de exposición al virus fue por contacto familiar en domicilio y el no uso correcto de un cubre boca. (17)

Como la diabetes mellitus y la hiperglicemia pueden conducir a un mayor riesgo de infección secundaria y mortalidad, se sugiere que el manejo de glucemia debe valorarse y optimizarse mejor,
ISSN 2773-7705

Periodo. Enero - Junio 2020

Vol. 2, Nro. 1, Publicado 2020-06-30

por lo que se recomienda realizar control glicémico, comparado a nuestro resultado el control fue ineficiente alterando los valores glicémicos, a pesar que la medicación no se cambió ni se suspendió.

Hernández et al, muestra que el síndrome post Covid, repercute en la mayoría de la población general y en particular sobre los grupos vulnerables por lo que considera seguimiento con equipo interdisciplinario para disminuir las secuelas y la salud mental, La mayoría de nuestros adultos encuestados, se encuentra estable con control médico, otros sin control, y un 16\% de la población falleció a causa de complicaciones graves y falta de espacio físico en UCI. (18)

\section{Conclusiones}

Se llega a concluir que:

- Los factores asociados a complicaciones y a enfermedad por Covid-19 en adultos mayores diabéticos de la ciudadela Parrales Y Guale de la ciudad de Jipijapa fueron la comorbilidad asociada a la diabetes, la más común es la hipertensión arterial, la edad también juega un papel importante que se convierten en un componente de riesgo, el contacto familiar o visitas domiciliarias como factor importante para la exposición a la enfermedad, los factores emocionales por preocupación por la pandemia y los valores reflejados de la glicemia contribuyen a una frecuencia elevada en pacientes con las patologías investigadas.

- Se identificó dentro de la población de referencia y de los casos, que los marcadores de riesgo asociados a mayor frecuencia de afectación, es en mujeres en comparación a los hombres y la edad más representativa de la muestra va desde los 65 a 75 años.

- Los factores biológicos- socio sanitario con notable preponderancia es la sintomatología leve presentada con mayor frecuencia en el grupo de investigación, se recalca que existió un $17 \%$ de pacientes con síntomas graves; el abandono de tratamiento, el acceso a una atención de salud pública, los hábitos alimenticios son importantes al momento de establecer el pronóstico del paciente con estas dos enfermedades asociadas. 
Referencias

1. Aquino-Canchari CR, Quispe-Arrieta dC, Huaman Castillon M. revista Habanera.de Ciencias Médicas. [Online].; 2020. Available from: http://scielo.sld.cu/scielo.php?script=sci_arttext\&pid=S1729$519 \times 2020000400005$.

2. Pacheco DVM. PROBLACIONES VULNERABLES Y EN SITUACIÓN DE VULNERABILIDAD. Red ALAC de Comisiones Nacionales de Bioetica en Salud - UNESCO, Comisión Nacional de Bioética en Salud; 2018.

3. Wenzel JEyRP. Un nuevo coronavirus emerge. Elsevier Public Health. 2020 Febrero 13; 220.

4. Crespo RM, Crespo M. Pandemia COVID-19, la nueva emergencia sanitaria de preocupación internacional. SEMERGEN. 2020 Agosto; 46.

5. Gustavo B. De Wuhan a Luján. Evolución espacial del COVID-19. POSICION. 2020 Abril; ISSN 2683$8915[$.

6. Lemus-Delgado PAE\&D. El actuar del Dr. Li Wenliang ante el brote del COVID-19 a la luz del principio de beneficencia. Veritas. 2020 Agosto; 46.

7. Mesa M. Riesgo globales y multilateralismo: el impacto de la Covid 19. In. Madrid; 8va edición. p. 4041.

8. Haro AS, Palacios Calderón E. Caracterización epidemiológica de Covid-19 en Ecuador. [Online].; 2020. Available from: https://www.iajmh.com/iajmh/article/view/99/110.

9. Gerardo Patricio Inca Ruiz AIL. Evolución de la enfermedad por coronavirus (COVID-19) en Ecuador. revistas Spoch. 2020 Abril; 11(1).

10. Organization PAH. Diabetes. AOnline]. Available from: https://www.paho.org/hq/index.php?option=com_content\&view=category\&id=4475\&layout=blog\&Ite mid=40610\&lang=es\&limitstart=15\#: :text=La\%20diabetes\%20de $\% 20$ tipo $\% 202$, excesivo $\% 20$ y $\% 20 \mathrm{a}$ $\% 201 \mathrm{a} \% 20$ inactividad\%20f\%C3\%ADsica.

11. Yan Zheng SHLFBH. Nat Rev Endocrinol. [Online].; 2018 Feb. Available from: https://www.intramed.net/contenidover.asp?contenidoid=94710\#: :text=A\%20nivel\%20mundial\%2C $\% 20$ aproximadamente $\% 201$,esta\%20epidemia\%20mundial\%20de\%20DM2.

12. Mehta DR. Diabetes va en alza en América Latina y el Caribe. [Online].; 2018. Available from: https://clustersalud.americaeconomia.com/sector-publico/la-diabetes-va-al-alza-en-america-latina-y-elcaribe.

13. Ecuador MdS. Ministerio de Salud: prevención y autocuidado son claves para controlar la diabetes. [Online].; 2018. Available from: https://www.salud.gob.ec/ministerio-de-salud-prevencion-yautocuidado-son-claves-para-controlar-la-diabetes/.

14. Ecuador MdSPd. CONSENSO DE RECOMENDACIONES PARA EL TRATAMIENTO DEL COVID19 EN PERSONAS ADULTAS MAYORES. [Online].; 2020. Available from: https://www.salud.gob.ec/wp-content/uploads/2020/09/Recomendaciones-para-el-tratamiento-delCovid-19-en-personas-Adultas-Mayores.pdf. 
15. Ebrahimzadeh BA. Prevalencia de comorbilidades en pacientes con COVID 19. [Online].; 2021. Available from: https://www.ncbi.nlm.nih.gov/pmc/articles/PMC7296605/.

16. Enfermedad por coronavirus C1. Centro de Coordinación de Alertas y Emergencias Sanitarias. [Online].; 2021. Available from: https://www.mscbs.gob.es/profesionales/saludPublica/ccayes/alertasActual/nCov/documentos/ITCoron avirus.

17. Greenhalgh T BSM. Face masks for the public during the covid-19 crisis. [Online].; 2020. Available from: https://www.bmj.com/content/369/bmj.m1435.

18. Rodríguez H. Medicentro Electrónica Scielo. [Online].; 2020. Available from: http://scielo.sld.cu/scielo.php?script=sci_arttext\&pid=S1029.

19. OMS. Organización Mundial de la Salud. [Online].; 2017. Available from: https://www.who.int/features/factfiles/obesity/es/.

20. surgery If. Innovation for surgery. [Online].; 2020. Available from: https://www.midmed.com/es/surgery/comprendiendo-la-obesidad/.

21. OMS. Organización Mundial de la Salud. [Online].; 2016. Available from: https://www.who.int/features/factfiles/obesity/es/.

22. Primicias DD. Diario Digital Primicias. [Online].; 2019. Available from: https://www.primicias.ec/noticias/sociedad/sobrepeso-obesidad-muerte-alimentos/.

23. Madrid CMydRP. Centro Médico y de Rehabilitación Premium Madrid. [Online].; 2020. Available from: https://rehabilitacionpremiummadrid.com/blog/sara-carnicero/la-importancia-de-la-actividad-fisica-enla-prevencion-y-el-tratamiento-de-la-obesidad-y-sobrepeso-en-adultos-parte-

i/\#: :text=Adem\%C3\%A1s\%20de\%20lo\%20comentado\%20hasta,arterial\%2C\%20la\%20prevenc. 